\title{
James Webb Space Telescope Integrated Science Instrument Module Calibration and Verification of High-Accuracy Instrumentation to Measure Heat Flow in Cryogenic Testing
}

\author{
Brian Comber ${ }^{1}$ and Stuart Glazer ${ }^{2}$ \\ 'Orbital Sciences Corporation, Greenbelt, Maryland, USA, 20770 \\ ${ }^{2}$ NASA/Goddard Space Flight Center, Greenbelt, Maryland, USA, 20771
}

\begin{abstract}
The James Webb Space Telescope (JWST) is an upcoming flagship observatory mission scheduled to be launched in 2018. Three of the four science instruments are passively cooled to their operational temperature range of $36 \mathrm{~K}$ to $40 \mathrm{~K}$, and the fourth instrument is actively cooled to its operational temperature of approximately $6 \mathrm{~K}$. The requirement for multiple thermal zones results in the instruments being thermally connected to five external radiators via individual high purity aluminum heat straps. Thermal-vacuum and thermal balance testing of the flight instruments at the Integrated Science Instrument Module (ISIM) element level will take place within a newly constructed shroud cooled by gaseous helium inside Goddard Space Flight Center's (GSFC) Space Environment Simulator (SES). The flight external radiators are not available during ISIM-level thermal vacuum/thermal testing, so they will be replaced in test with stable and adjustable thermal boundaries with identical physical interfaces to the flight radiators. Those boundaries are provided by specially designed test hardware which also measures the heat flow within each of the five heat straps to an accuracy of $<2 \mathrm{~mW}$, which is less than $5 \%$ of the minimum predicted heat flow values. Measurement of the heat loads to this accuracy is essential to ISIM thermal model correlation, since thermal models are more accurately correlated when temperature data is supplemented by accurate knowledge of heat flows. It also provides direct verification by test of several high-level thermal requirements. Devices that measure heat flow in this manner have historically been referred to as "Q-meters". Perhaps the most important feature of the design of the JWST Q-meters is that it does not depend on the absolute accuracy of its temperature sensors, but rather on a difference in heater power, for which a table is empirically developed during a calibration campaign in a small chamber at GSFC. This paper provides a brief review of Q-meter design, and discusses the Q-meter calibration procedure including calibration chamber modifications and accommodations, handling of differing conditions between calibration and usage, the calibration process itself, and the results of the tests used to determine if the calibration is successful.
\end{abstract}

\section{Introduction}

A. James Webb Space Telescope and the Integrated Science Instrument Module (ISIM)

The James Webb Space Telescope (JWST) is an upcoming flagship observatory mission designed to be launched $\mathcal{I}$ into orbit around the second Lagrange point before 2018. It has a $6.5 \mathrm{~m}$ (deployed) multi-faceted mirror, and its science instruments operate in short to medium wavelengths to enable insights into the formation of the galaxies and

${ }^{1}$ James Webb Space Telescope ISIM Thermal Test Analyst

${ }^{2}$ James Webb Space Telescope ISIM Thermal Systems Lead, Thermal Engineering Branch, Code 545.3 
planets following the "Big Bang". NASA's Goddard Space Flight Center (GSFC) is the mission lead, and is directly responsible for the Integrated Science Instrument Module (ISIM), including its integration and test. The suite of four science instruments, located in the cryogenic volume behind the primary reflector (denoted as Region 1 in Figure 1-1), is comprised of the Near Infrared Spectrograph (NIRSpec), primarily sponsored by the European Space Agency (ESA) with substantial NASA contribution; the actively cooled Mid Infrared Instrument (MIRI), jointly sponsored by ESA and the European Consortium (EC); the Fine Guidance Sensor (FGS), provided by the Canadian Space Agency (CSA); and the Near Infrared Camera (NIRCam), provided by NASA. In flight, NIRSpec, NIRCam, and FGS are cooled to the range of $36 \mathrm{~K}$ to $40 \mathrm{~K}$ passively via heat transfer through complex high-purity aluminum thermal links to dedicated radiators, while the MIRI is actively cooled to approximately $6 \mathrm{~K}$ using a cryocooler. NASA provides the cooler and supplemental hardware for MIRI. The cooler's compressor is located in the ambient temperature spacecraft bus, outside the cryogenic Region 1. The science instruments are mounted on the ISIM structure, a special composite truss structure designed for exceptional optical stability, provided by NASA. The Observatory Prime Contractor is Northrop Grumman Aerospace Systems (NGAS).

Figures 1-1 and 1-2 illustrate the observatory and the major components of the ISIM, respectively. The ISIM Electronics Compartment (IEC), in close proximity to Region 1, houses the instrument and detector control electronics, and operates at approximately ambient temperature and is denoted as "Region 2". The ISIM Harness Radiator (IHR), which operates below 80K and is also provided by ISIM, rejects most harness parasitic heat loads to space before the harnesses enter Region 1. The ISIM thermal control subsystem provides 93 flight housekeeping temperature sensors on the instruments, ISIM structure, radiators, and other locations, and trim heaters to guarantee minimum instrument operational temperatures. It arranges for control of contamination control heaters on each instrument (except the MIRI), to avoid contamination during post-launch cool-down, and in the event of contamination on-station.

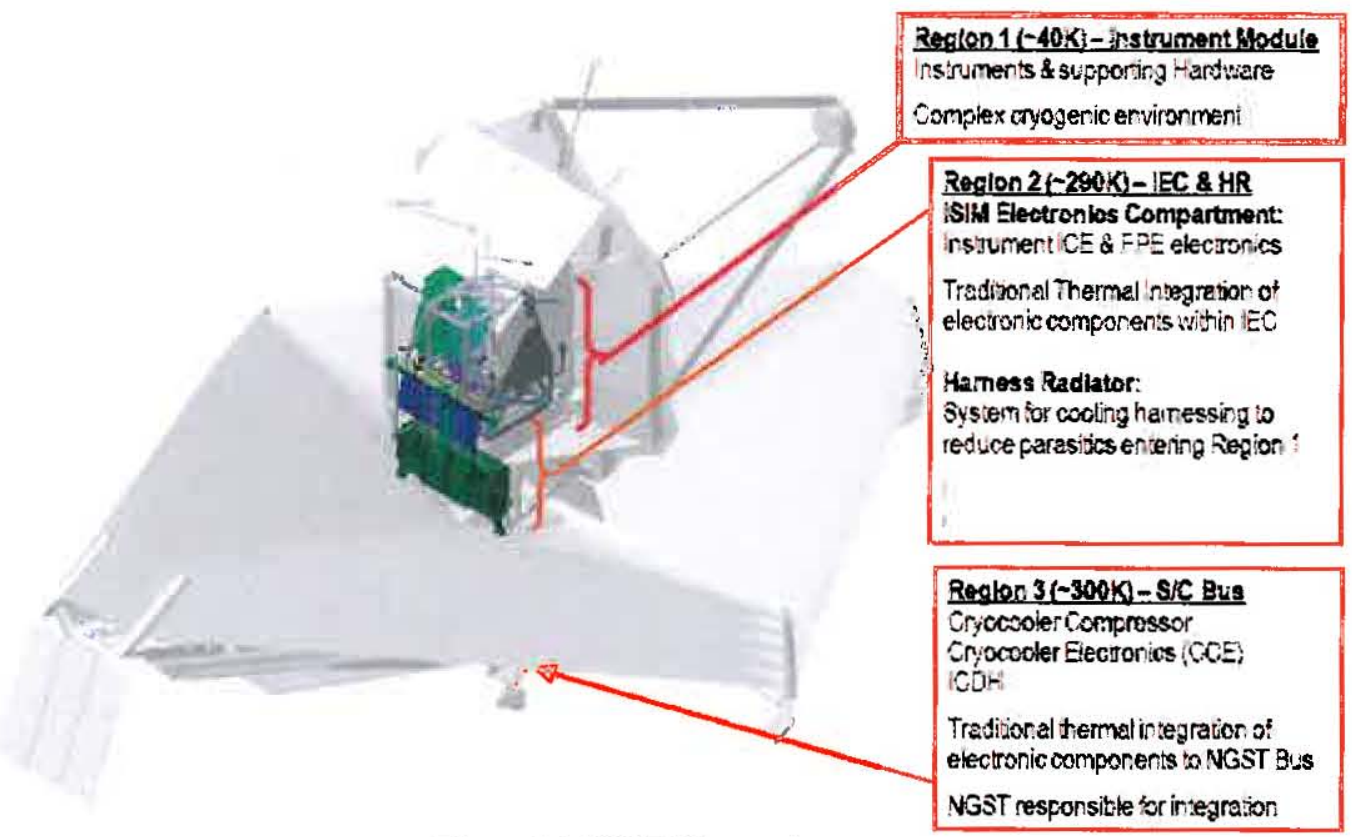

Figure 1-1 JWST Observatory 


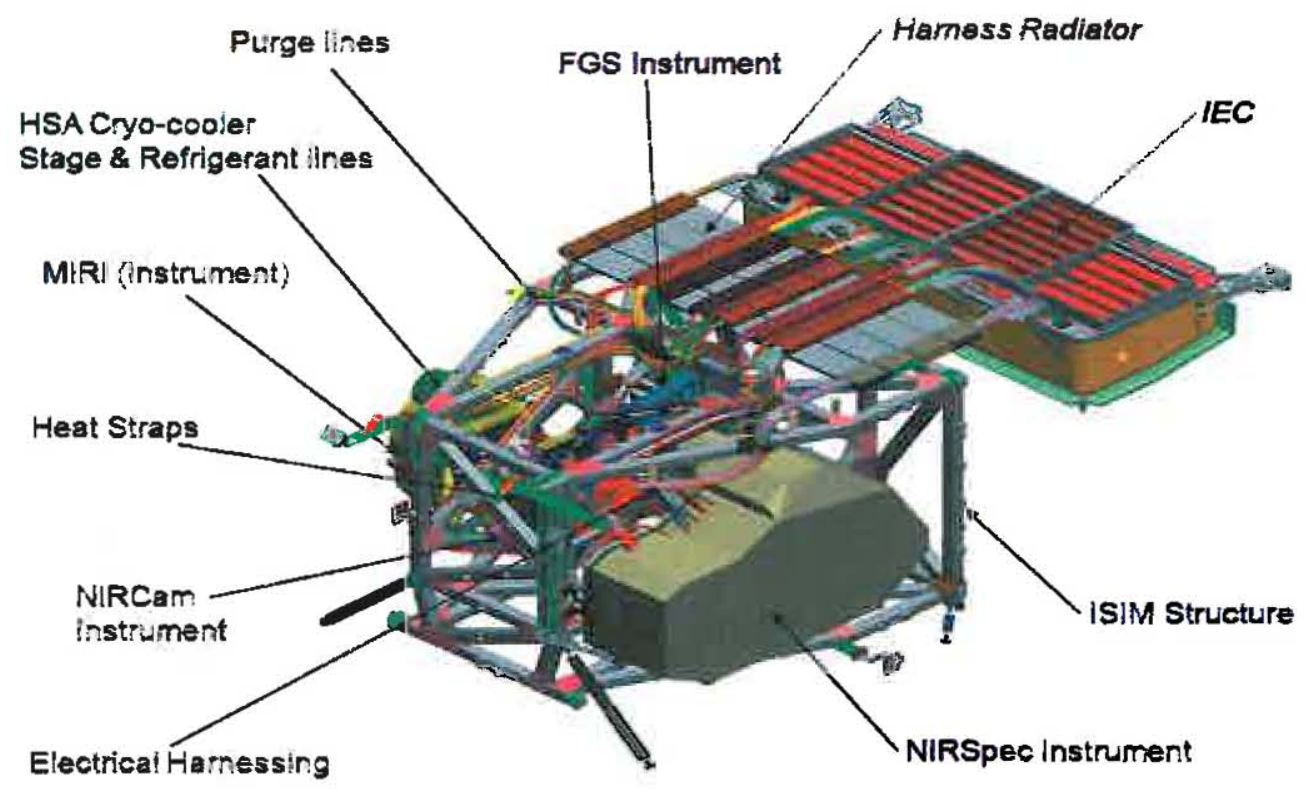

Figure 1-2 Integrated Science Instrument Module, with Harness Radiator and Integrated Electronics Compartment

Reference papers 1, 2 and 3 provide a more comprehensive description of the JWST and ISIM.

\section{B. Q-Meter Description and Purpose}

A Q-meter is a device that measures heat flow through it, and by extension, heat flow through anything that is attached to the device in series. In the simplest sense, a Q-meter can be thought of as a calibrated thermal conductor.

The ISIM element will be thermally tested in the Goddard Space Flight Center's Space Environment Simulator (SES) chamber in a 3-part thermal vacuum / thermal balance test, beginning in February 2013. Flight-like thermal boundaries will be provided by temperature-controlled radiative panels and conductive interfaces. The flight radiators, which provide the thermal boundaries at the ends of the heat straps, are not present in the ISIM-level thermal test; instead, the heat straps will terminate at the non-flight Q-meters. These Q-meters thus serve a dual purpose: 1) to provide stable and controllable thermal boundaries simulating the radiators, and 2) to measure with high accuracy the heat flow to the radiators.

The primary objectives of the ISIM Cryo-Vac test include:

- Verification of optical performance using a sophisticated optical source simulator situated below the ISIM;

- Verification of electrical and software operation;

- Qualification of the ISIM over its operational temperature range;

- Correlation of the thermal model by running several thermal balance cases, enabling accurate thermal predictions for flight.

- Validation of the ISIM thermal design

Figure 1-2 shows the high purity aluminum heat straps used to transfer heat from 3 of the 4 instruments to external radiators to passively cool them to their operational temperature ranges. The $5^{\text {th }}$ heat strap, attached near the MIRI mount feet and harnesses, reduces parasitic heat loads to the instrument, enabling the cryo-cooler to cool that instrument to $6 \mathrm{~K}$.

Measurement of heat transferred to the radiators under operational conditions is critical to ISIM thermal verification and model correlation. This is accomplished by the highly accurate Q-meters, since the heat straps are the principal avenue for the conductive heat transfer from the instruments to the radiators. The thermal model can be correlated using the large number of flight temperature sensors; however, accurate measurement of the heat flows to 
the radiators through the heat straps enables a more comprehensive understanding of the parasitics and test-induced heat leaks. The Q-meters, in series with the straps, provide this measurement capability. Naturally, with a system of this complexity, the Q-meter design is much more than simply a calibrated thermal conductor. The authors' earlier paper, "James Webb Space Telescope Integrated Science Instrument Module Design, Optimization, and Calibration of High-Accuracy Instrumentation to Measure Heat Flow in Cryogenic Testing" (Reference 4), describes the design of the Q-meters in detail. This paper reports on the methods, set-backs, results, and lessons learned during the Qmeter calibration testing performed at Goddard Space Flight Center (GSFC) in late 2011 and the first half of 2012.

\section{Calibration Test Set-Up and Challenges}

\section{A. Q-meter Design Overview}

By design, the Q-meter is completely reliant on an extensive calibration campaign in order to make an accurate measurement of heat flow in a thermal vacuum test. Figure 2-1 illustrates how the Q-meters work. The basic design incorporates an Aluminum 6061-T6 "spool" turned from a single piece of Aluminum. There are three "bulk masses", or stages, where the temperature is controlled. In Figure 2-1, they are labeled "A" for the top, "B" for the middle, and "C" for the bottom. At location C, the Q-meter is bolted to its thermal sink. This could be a cold plate with refrigerated helium pipes, or in the case of the calibration test, a copper bracket attached to a cryocooler. Location B is temperature-controlled via PID heaters. Aluminum 1100 shields bolt to Location B and cover 5 sides of the Q-meter without touching any other surfaces. Heaters at Location B enable this stage to overcome variable external environment heat loads, and provide a stable and completely known thermal environment for the upper stage, Location A. Thus, only precise knowledge of temperature at Location "B" is required. For Location A, however, both the temperature and the heater power are important. For each " $\mathrm{A}$ " setpoint temperature, a corresponding " $\mathrm{B}$ " setpoint temperature is defined to maximize accuracy and minimize total heater power that must be absorbed by the refrigerator or cryo-cooler.

Baseline Q-meter calibration is performed without external heat strap loads to measure the heater power required at Location $\mathrm{A}$ to maintain the specified temperature there, given the specified temperature at Location $\mathrm{B}$. The total power required at Location " $\mathrm{A}$ " to hold its temperature at a specified value is constant, so that when a strap is connected, reduction in Stage " $A$ " heater power from the baseline represents the external heat from the strap.

The absolute accuracy of the CERNOX sensors used in locations " $A$ " and " $B$ " is approximately $+/-20 \mathrm{mK}$ at operating temperature. After calibration, however, the accuracy/repeatability of the difference in temperature across the calibrated neck is what is important. GSFC has been advised by the sensor manufacturer that thermal drift over time can be reduced if the sensors are not subjected to multiple rapid thermal cycles. Additionally, since the sensors on both stages are taken from the same chip, the likely scenario is that the two sensors will drift in the same direction, maintaining a more accurate measurement of the difference in temperature. Given these predictions, thermal drift of the sensors translates into an expected long term repeatability of heater power and accuracy of the Q-meter, to less than $+/-2 \mathrm{~mW}$ over the entire test program.

Figure 2-2 shows the detailed design that results from the concept shown in Figure 2-1. Figure 2-3 shows the Qmeter on the laminar flow bench before final integration with the shields and top heater plate. Some of the built-in features of the Q-meter which contribute to the robustness of the design are visible in Figure 2-3. The shields are attached to the middle level only, in order to maintain a constant and repeatable radiative environment for the calibrated top neck (A to B). The thermal contacts are enhanced using Apiezon thermal interface material. All of the very thin phosphor bronze wires are staked to the Q-meter spool in multiple locations with stycast, such that they become part of the calibrated and repeatable Q-meter. Finally, all of the heaters are installed on "spreader plates" made of Aluminum 1100 in order to evenly spread the heat before it is transferred into the Aluminum 6061 spool. 


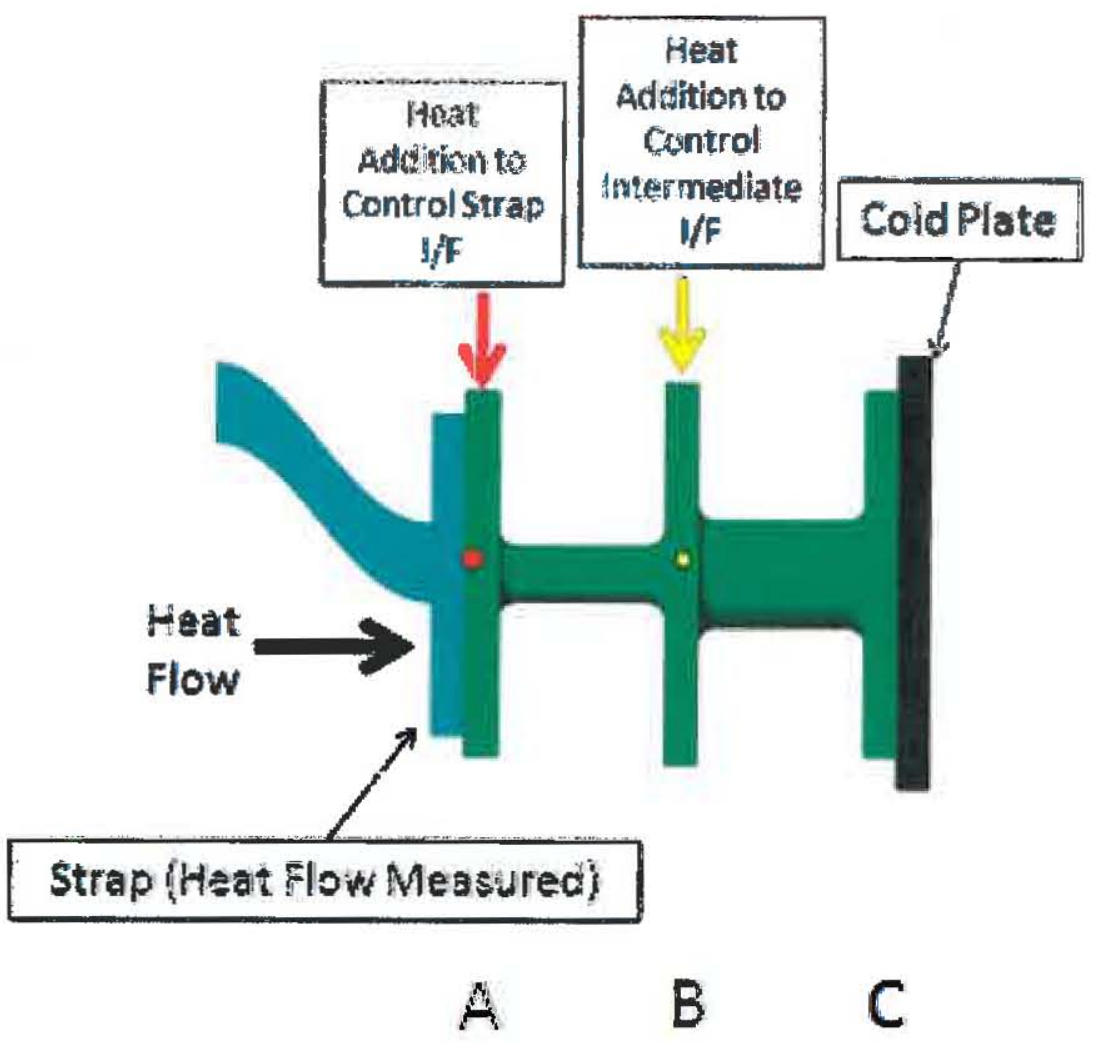

Figure 2-1 Q-Meter Basic Operation

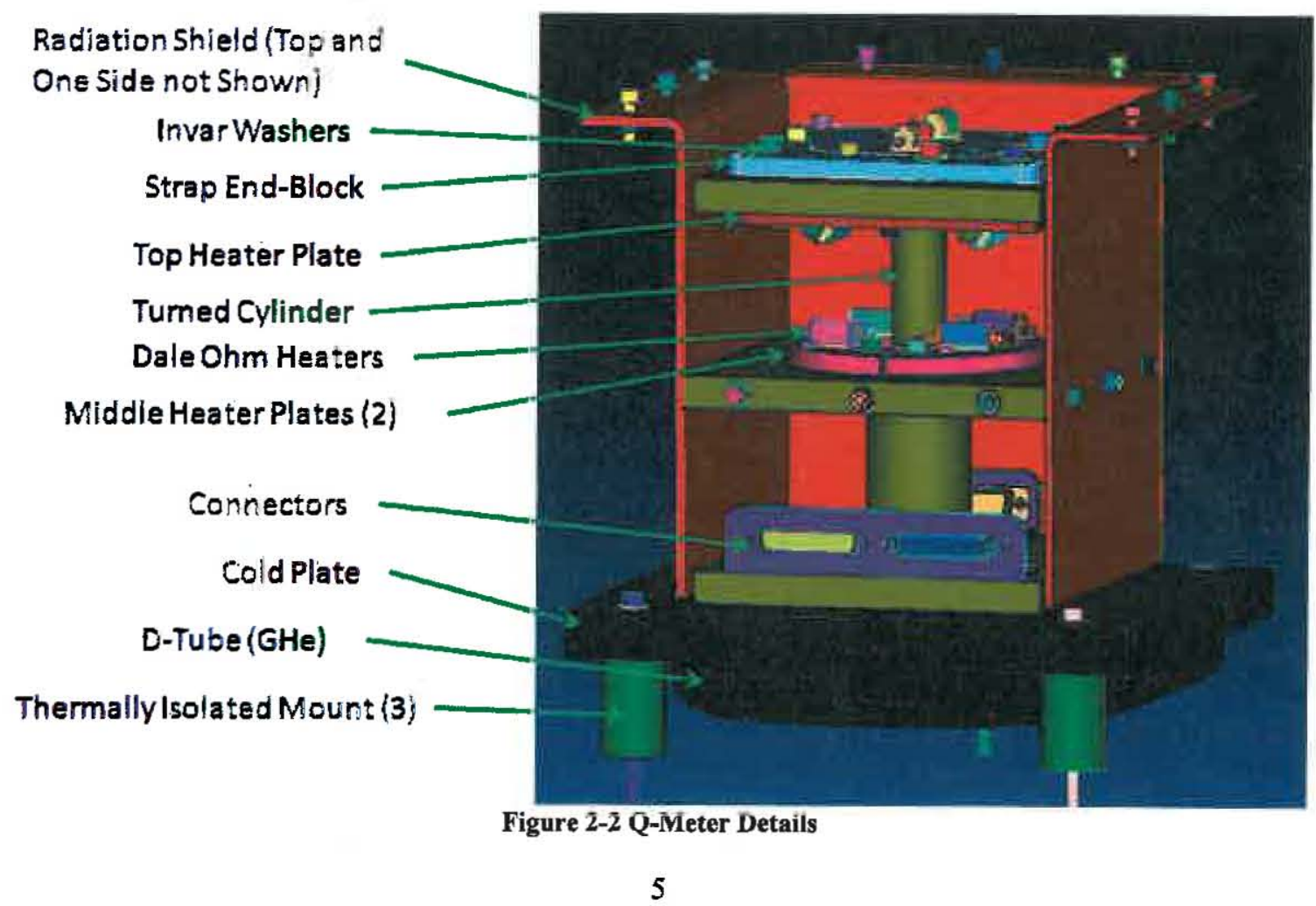

American Institute of Aeronautics and Astronautics 


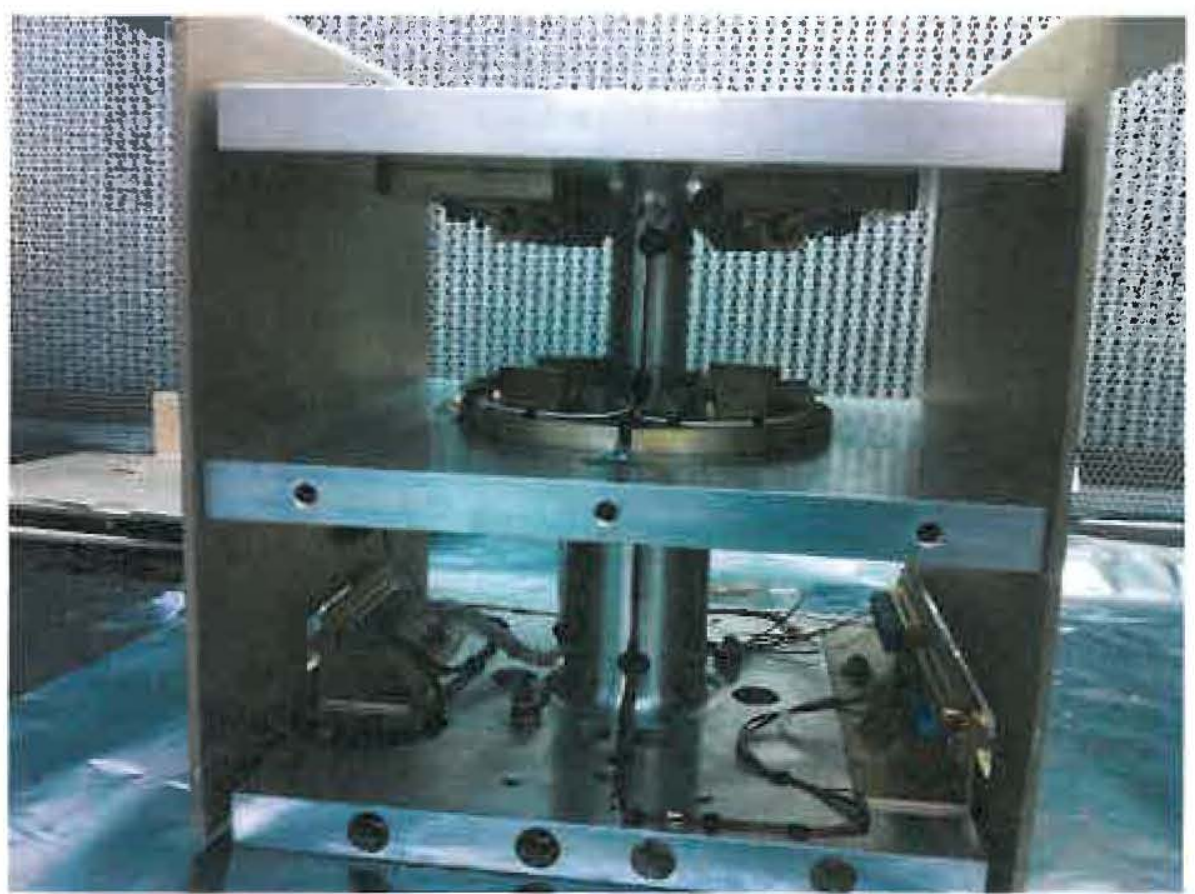

Figure 2-3 Q-Meter Assembly Photograph

\section{B. Requirements and Goals for the Calibration Program}

The requirements of the Q-meter Calibration test campaign are to verify its key design features, and to create a table of temperature setpoint vs. top heater power for use in calculating strap heat flow in later testing with additional hardware. The Q-meter calibration test should:

1. Report heater power over a large range of set-points, and show that it is within acceptable parameters for use in the ISIM Cryovac tests.

2. Measure the conductance vs. temperature of the neck from Location $A$ to Location $B$ in case measurements are required at temperatures other than the specifically calibrated points.

3. Demonstrate that the Q-meter measurement is repeatable after multiple thermal cycles.

4. Demonstrate that the Q-meter is insensitive to variations in both conductive and radiative environment.

5. Prove that the Q-meter actually measures heat flow to $+/-2 \mathrm{~mW}$ over the range of provided boundary temperatures (Stage "A") of $18 \mathrm{~K}$ to $50 \mathrm{~K}$.

\section{Test Set-Up}

The Q-meter calibration test utilizes a small Varian chamber at GSFC. Figure 2-4 shows the bottom half of the chamber before installation of the Q-meter. The chamber has a cryocooler that is capable of reaching approximately $12 \mathrm{~K}$ without load, and $20 \mathrm{~K}$ with $5 \mathrm{~W}$. The cold-head is shown in the center of the picture, with a circular bolt pattern. The Q-meter is attached via a copper bracket to the cold-head. The chamber also features a nitrogen shroud, controllable from room temperature to $100 \mathrm{~K}$, that lowers onto the portion of the chamber shown in Figure 24 , along with the rest of the pressure vessel. Figure 2-5 is a wider view of the chamber with the Q-meter installed. Great lengths were taken to allow the Q-meter to get to operating temperature, and to prevent shorts between the harnessing and the Q-meter that might invalidate the calibration. In Figure 2-6, the large disc covering the portion of the chamber that does not have a nitrogen shroud is connected to the first stage of the cryocooler, and reaches about $50-60 \mathrm{~K}$. As is shown in Figure 2-6, all wires are sunk using aluminum tape to this stage first, when they enter the chamber from the room temperature pressure vessel walls. After allowing a span of wire large enough to avoid thermal shorting of stages, the wires are additionally sunk using copper tape to the copper bracket that is attached to the second stage of the cryocooler, and are then plugged into the Q-meter's external connectors. In order to avoid 
interference issues when closing the chamber, the nitrogen shroud does not fully extend into the bottom half of the chamber. It stops a few inches short of that point. Instead of using a blanket to shield the Q-meter from room temperature radiation, an aluminum tape barrier is utilized (Figure 2-7). Unlike blankets, which will warm in response to room temperature radiation on one side, the aluminum tape immediately conducts the heat into the nitrogen shrouds. Finally, an MLI blanket covers the entire bottom portion of the chamber, and an SLI blanket covers the Q-meter in the final configuration. Figure 2-8 shows the final configuration, during the chamber break at the end of the $3^{\text {rd }}$ cycle of the calibration test. The blanket and the aluminum tape ring nicely conform to each other and complete the isolation of the Q-meter from the ambient environment.



Figure 2-4 Empty Chamber 


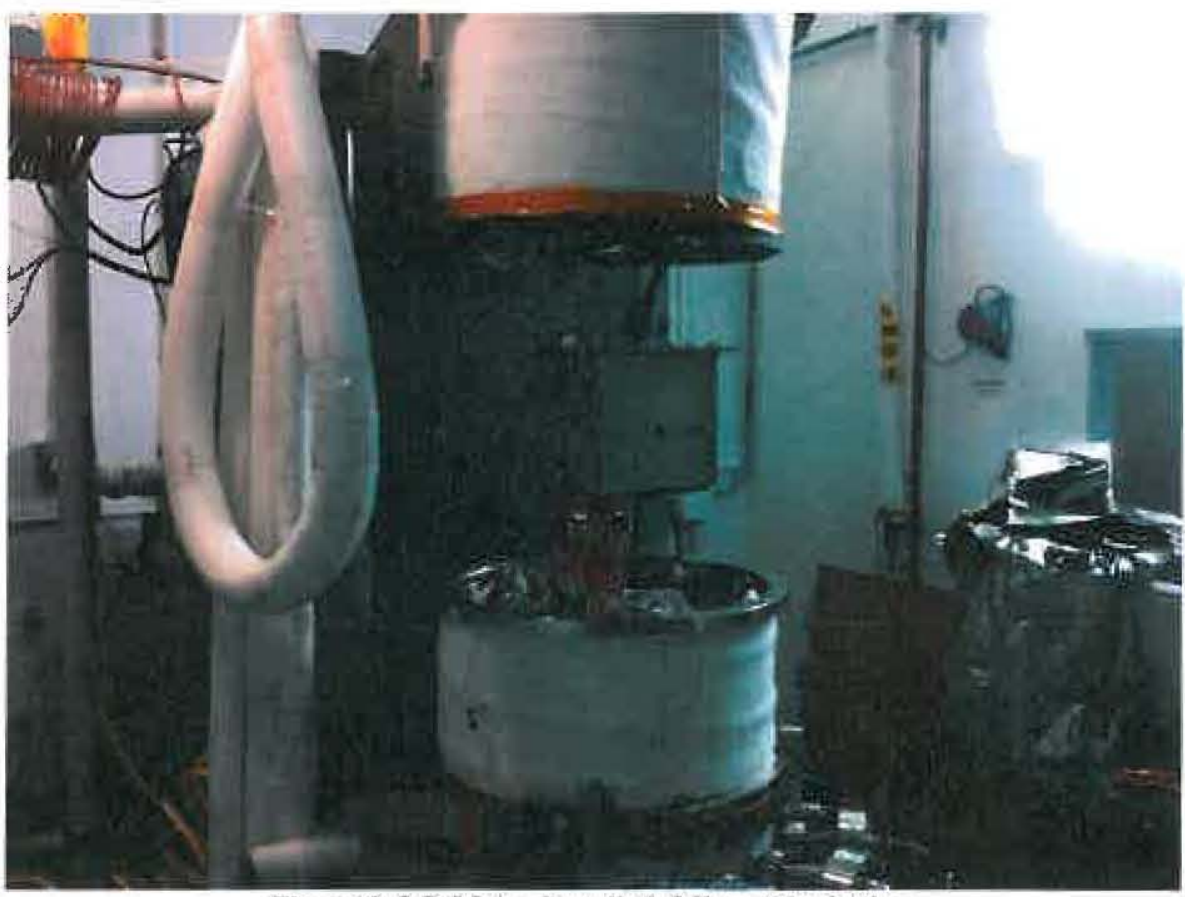

Figure 2-5 Q-Meter Installed, Minus Blanketing

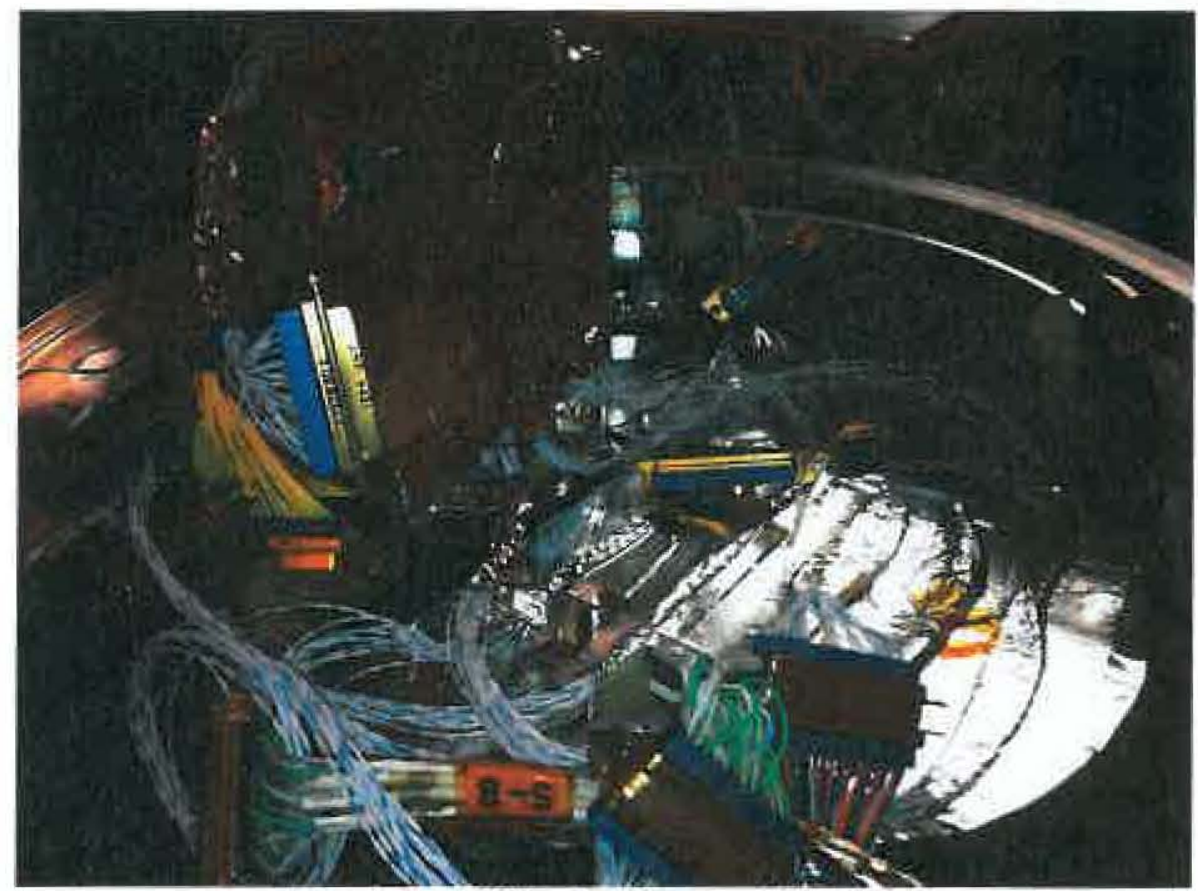

Figure 2-6 Chamber Harness Sinking 


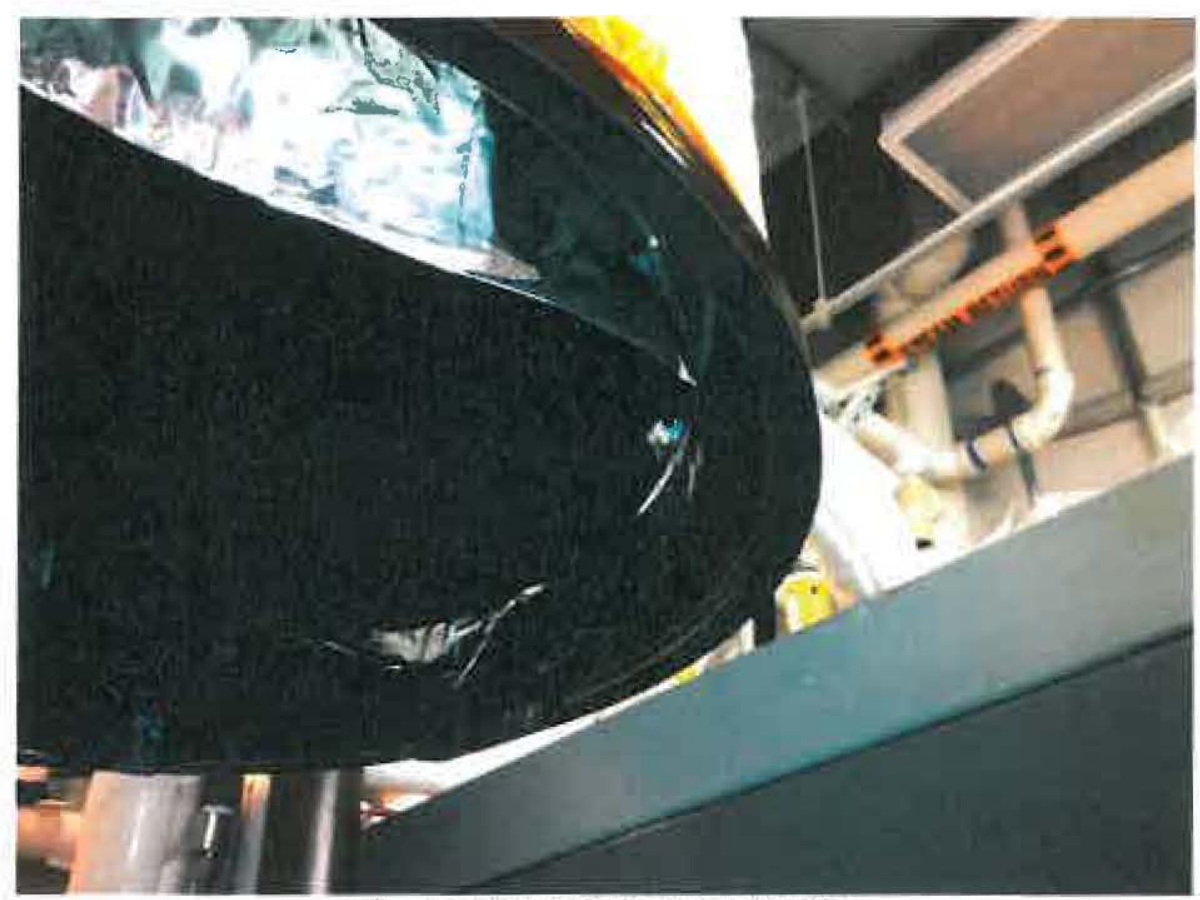

Figure 2-7 Nitrogen Shroud Closeout

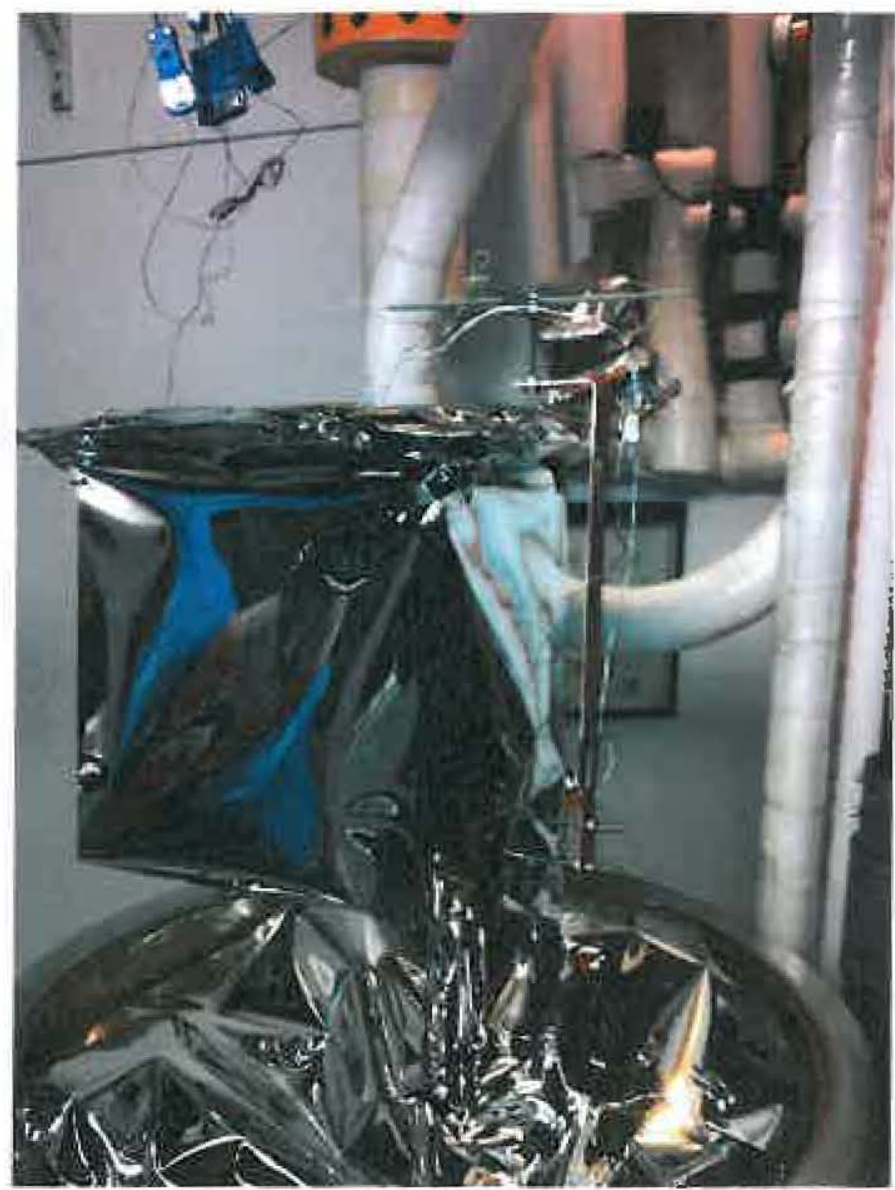

Figure 2-8 Q-Meter Fully Blanketed, During Chamber Break before Cycle 4 
Figure 2-8 shows the Q-meter with a wire protruding from the top, which is suspended from an isolated bracket, and finally sunk to the second stage of the cryocooler. That wire is only connected when the heater calibration plate (not part of Q-meter) is used to check the accuracy of the Q-meter after the baseline calibrations. The calibration plate has heaters that simulate the footprint of the flight heat straps in the Cryovac test. The wires for those heaters and sensors are coiled inside and taped to the plate such that they cannot touch the top shield throughout the initial baseline calibration. When used to verify Q-meter calibration accuracy, the wires are pulled out, suspended as shown in Figure 2-8 and Figure 2-9, and attached to power sources. A known amount of heat is then applied at the heater calibration plate, and the Q-meter is checked to verify that the total heat (internal plus external) required to maintain Stage A temperature at the thermal boundary is identical to that required in the baseline calibration, without external heat..

The routing of the wires for this measurement poses a challenge. It is estimated that the un-sunk wire length would be approximately 10 " maximum, and a hand calculation for the conductive heat leak of the 28 gage phosphor bronze heater wires and the 36 gage phosphor bronze sensor wires indicates about $0.8 \mathrm{~mW}$ if the top of the Q-meter is set to a high temperature setpoint in the 40 's $\mathrm{K}$. Additionally, in the opposite direction, with $1 \mathrm{~W}$ to be measured on the calibration load heater plate, hand calculations show Joule heating of about $0.7 \mathrm{~mW}$ in the wire. Provisions for an external assembly to maintain an adiabatic wire interface were considered, but rejected because they would only have resulted in marginal improvement in measurement accuracy. The final design maximizes the unsupported length of the wire, minimizing parasitic heat leak to the heater plate. Additionally, during the final cycle of the calibration, only $200-300 \mathrm{~mW}$ is applied at the heater plate to better match actual expected test values, and to reduce Joule heating in the wires.

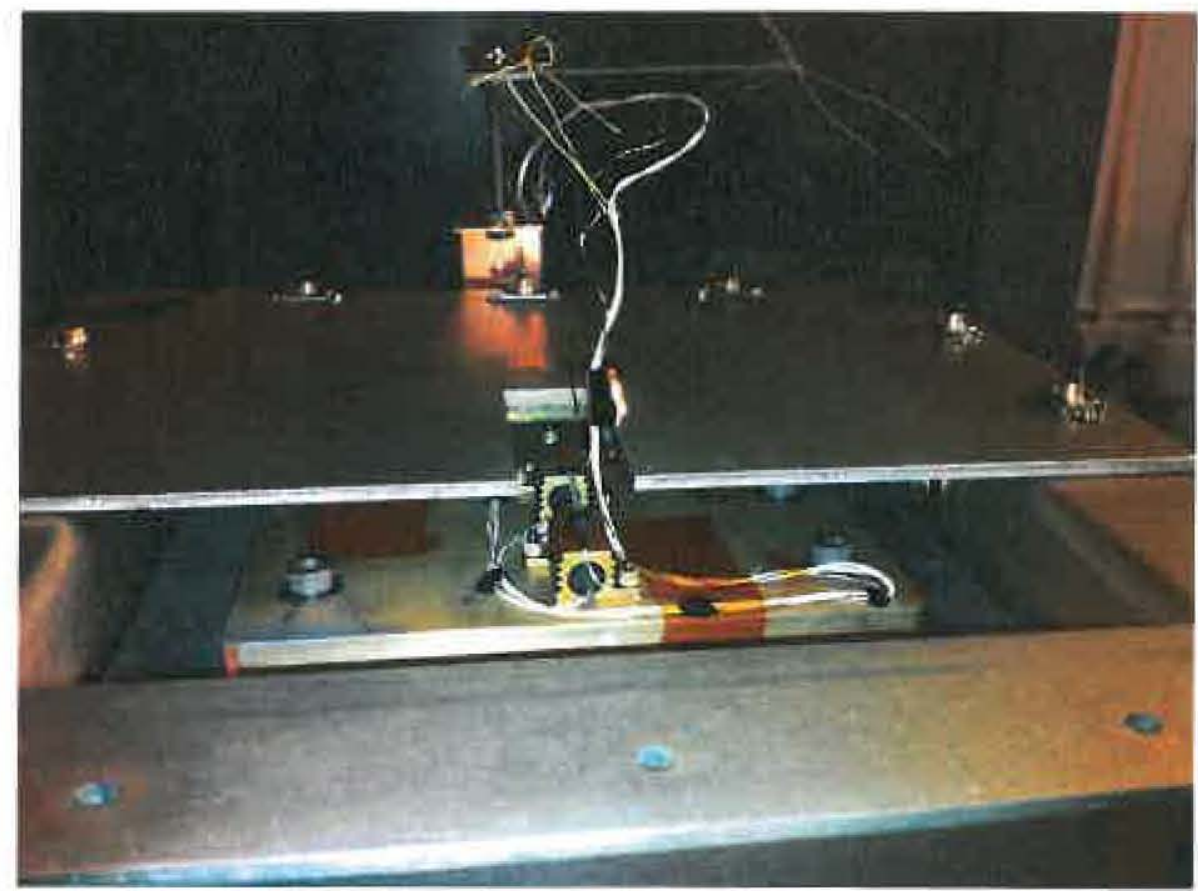

Figure 2-9 Calibration Load Heater Plate

\section{Calibration Test Profile}

Calibration test goals described in section B are met with a calibration program including 4 thermal cycles (ambient to cryo), with up to 162 thermal balances. Each balance point requires approximately 1 hour, because the Q-meter mass is approximately $5 \mathrm{~kg}$, and practically exclusively made of aluminum. The complete calibration program (4 thermal cycles) requires between 1.5 and 2 weeks per Q-meter. Calibration of the Q-meter during the first three cycles is completely automated, but the final cycle requires a chamber break and user input during postchamber break cryo balances. 
Figure 2-10 shows the planned calibration test profile, annotated with descriptions of the test phases. The first phase, after the initial cool-down fulfills goal \#1 from the list in the previous section. The result of those 55 temperature setpoints is a look-up table with 110 pairs of setpoints for the top (A) and middle (B) Q-meter levels. Since the Q-meter needs to be a robust instrument for 3 tests, each of which last 3 months, redundant heaters and temperature sensors are built into the system. Therefore, for each temperature setpoint, a balance must be completed for the A side heaters and sensors, and also for the B side heaters and sensors. The second "phase" of the testing fulfills goal \#2 from the list above. After the initial population of the Q-meter calibration matrix, the middle and top setpoints are set only $1 \mathrm{~K}$ apart, and "walked down" in $1 \mathrm{~K}$ increments across the expected useful range of the Qmeter. Setpoints with $2 \mathrm{~K}$ difference in temperature are mixed in, in order to rule out systematic errors in the measurement. This phase of the test quantifies the actual conductance of the calibrated neck between the temperature sensors. In the first phase, discrete setpoints are tested. The discrete setpoint method depends on choosing a calibration point that matches the final required temperature setpoints in the Cryovac test. Although the radiator temperature boundaries for the Cryovac balances are known at this point, they may change slightly as the Observatory thermal design and analysis proceeds. The 55 temperature setpoints in the calibration test span the range from $20 \mathrm{~K}$ to $50 \mathrm{~K}$ on the top of the Q-meter, and they are clustered around the current predictions for the radiator temperatures. Near those "sweet spots", the Q-meter is calibrated at intervals of just $0.25 \mathrm{~K}$. However, for setpoints between the "sweet spots", the Q-meter may only be calibrated at $1 \mathrm{~K}$ increments. Stepping through the temperature range with a IK DT between stage A and stage B will enable interpolation of heater power at Stage " $\mathrm{A}$ " to accommodate any changes to defined boundary temperatures.

After the conductance is quantified, the Q-meter is returned to ambient temperature and held at that level for approximately 4 hours. The second cycle fulfills goal \#3 (repeatability) in the list above. A small subset of the full calibration is performed during the second cold cycle and the results are compared against the results from the first cycle.

The third cycle is very similar, with the exception of the nitrogen shroud profile. The nitrogen shroud is set at $150 \mathrm{~K}$ in order to prove that the Q-meter shield and SLI make it insensitive to changes in the environmental temperature between its calibration and usage in a test. In the Cryovac test, while it is taking readings, the Q-meter will see an environment of about $50 \mathrm{~K}$ instead of $100 \mathrm{~K}$, but that exact scenario cannot be replicated in the calibration chamber. 


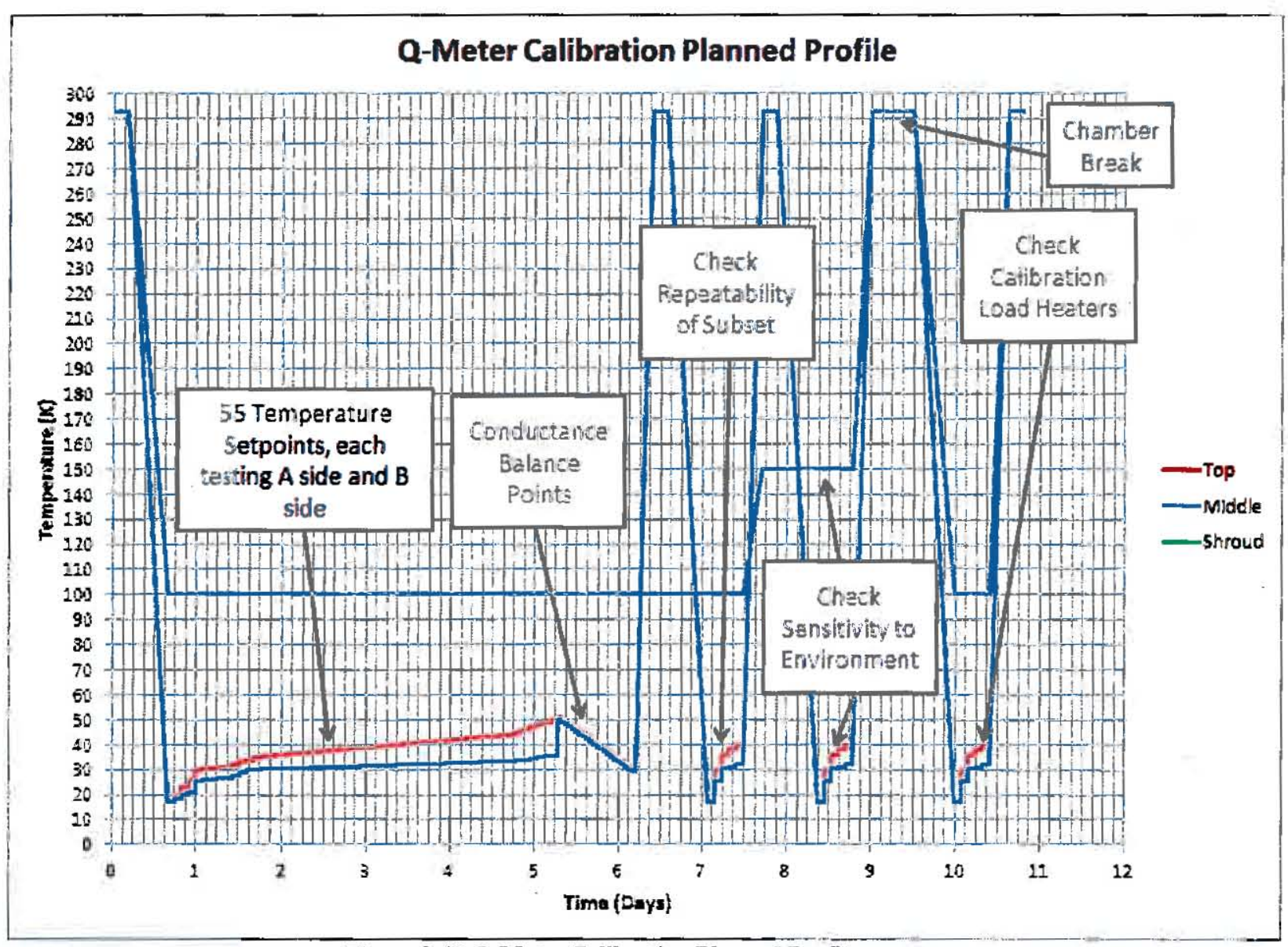

Figure 2-10 Q-Meter Calibration Planned Profile

After the third cold cycle, the Q-meter and chamber are returned to ambient temperature and a chamber break is performed. For the first three cycles, the wires for the calibration load heater plate are tucked inside the Q-meter. The wires themselves, and the small hole in the shield, could affect the Q-meter calibration by multiple $\mathrm{mW}$ if they penetrated the shield during the first three cycles. For the final cycle, the wires for the heaters and sensors on the top plate are suspended from an isolated structure above the Q-meter, and power is applied to the heaters to simulate heat arriving from the flight heat strap. The Q-meter's final test during calibration fulfils goal \#5 in the list above. Some inaccuracy from the conduction and Joule heating in the wires is expected, but the Q-meter should be able to back-calculate the amount of heat being applied to the load calibration heaters to nearly the accuracy expected of the Q-meter.

\section{E. Test Execution Challenges}

Challenges in the actual execution of the test arose in the complexity of the Labview system that controlled the test, and in the post-processing of the large volume of data that the calibration generates. Since the Q-meter is a non-flight instrument, it is acceptable to thermally test it without continual human attendance. The Labview program that runs on an external computer allows the user to input a large matrix of setpoints, and define how the system moves from one setpoint to another (control methodology, stability criteria, etc). After much fine-tuning, the Qmeter calibration test reliably set test heaters and monitored the stability of the top and middle (A and B) heaters. When the heater power becomes stable (measured by averaging a range of readings separated by multiple minutes), the system declares a 30 minute soak and then moves on to the next setpoint. It was also able to warm-up and cooldown on its own, such that the first three cycles could be performed without any interaction. 
The other early challenge in the calibration test presented itself in the interpretation of the volume of data being collected by the system. The system records temperature and heater information approximately every 2 seconds. Figure 2-11 shows the statistics performed on a set of 300 datapoints $(10 \mathrm{~min})$ for a particular temperature setpoint. This set of data is quite good, showing a slope of only $0.018 \mathrm{~mW} / \mathrm{hr}$ and a similarly small standard deviation and range of the data. The average of this data is the final result for the heater required for this timestep. Initially, these statistics were performed at the end of the step, in order to get the best answer. However, it was found that occasional disturbances in the cryocooler could affect the reading of the top heater power. Therefore, a routine was developed in Visual Basic for Applications that performed the statistics shown in Figure 2-11 for many time periods within the soak, and reported the results and location of the best set of $\mathrm{X}$ datapoints.

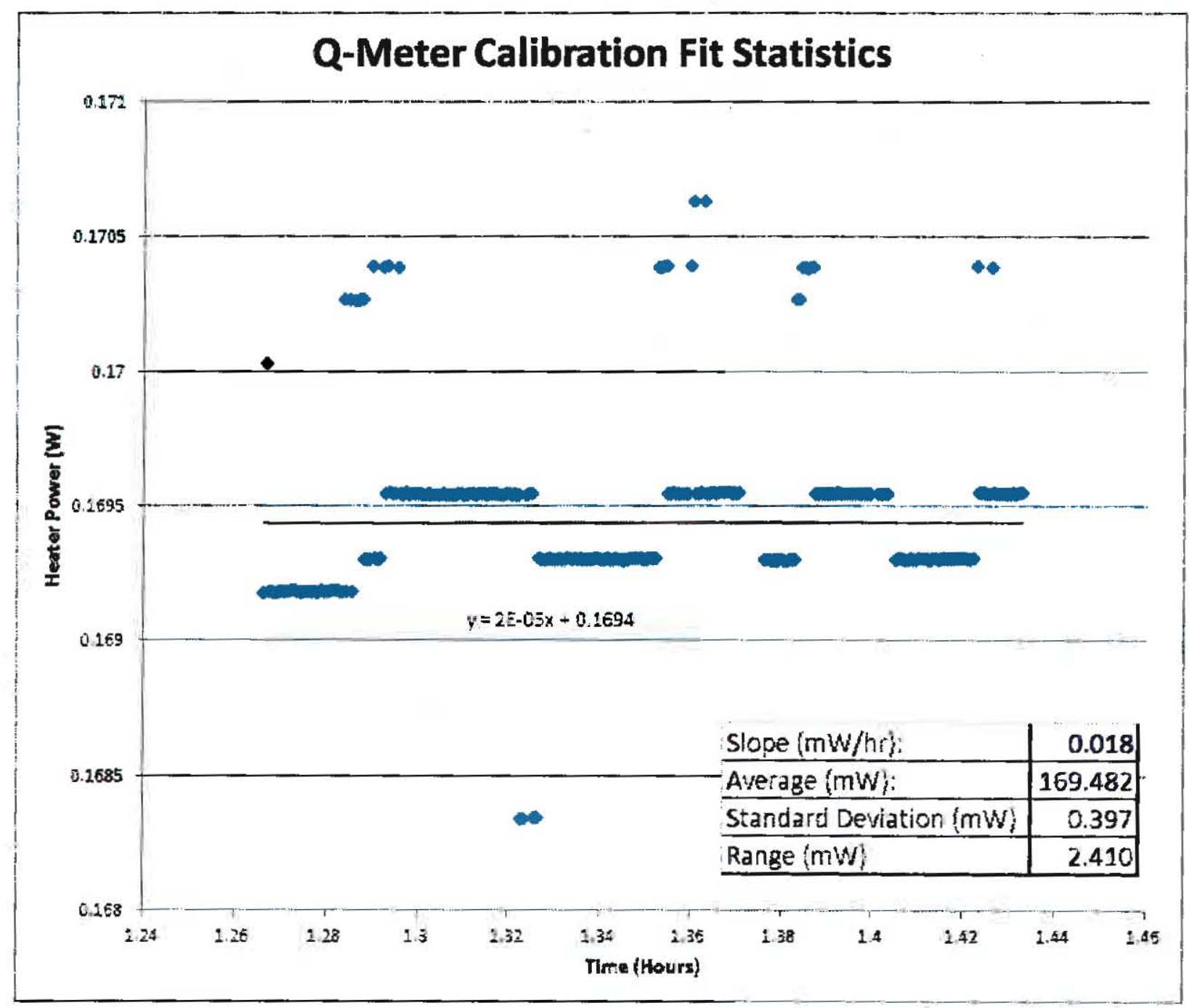

Figure 2-11 Q-Meter Calibration Statistics 


\section{F. Calibration Test Initial Results}

Figure 2-12 shows an early set of results, when 100 datapoints were chosen for the average instead of 300 . It shows a subset of the 55 temperature setpoints, for both primary and redundant heaters. Almost all of these results show good slope, standard deviation, and range of readings for the datapoints selected for statistics.

\begin{tabular}{|c|c|c|c|c|c|c|c|c|c|c|c|c|c|c|}
\hline \multirow[b]{2}{*}{ S:ês } & \multicolumn{5}{|c|}{ Monitor } & \multicolumn{5}{|c|}{ Bes: 100} & \multicolumn{4}{|c|}{ Lest 300} \\
\hline & TOPT & VIAST & 3ot T & Mid Q & 3012 & Sope & Average & S:cev & Range & Spo: & Siope & \begin{tabular}{|l|} 
Average \\
\end{tabular} & Stcev & Range \\
\hline $0 \mid A_{2}$ & 18 & 16.5 & 14.5 & 0.54 & 0.32 & 2.2 & 0.37979 & 20 & 90.3 & $89 \%$ & 4.9 & 0.377082 & 290 & 32 \\
\hline $2 i_{1}$ & 18 & 16.5 & 14.5 & 0.54 & 0.33 & 88 & 0,381779 & 0.3 & 3.2 & $90 \%$ & dis & $0.3813: 5$ & 324 & 6.1 \\
\hline $2\left(A_{i}\right.$ & 20 & 18 & 16 & 0.53 & 1.33 & $0 D$ & 0.565382 & (2.0) & 0.0 & 20036 & $\theta, 1$ & 0.561694 & 0.5 & $5, A$ \\
\hline $3 / 5)$ & 20 & 18 & 16 & 0.52 & 1.31 & 0.3 & 0.5627 & 0.2 & 5,4 & $56 \%$ & Q.5 & 0.560626 & 32 & 5,6 \\
\hline$A i A\rangle$ & 23 & 22 & 17 & 2.13 & $0.40^{\circ}$ & 0.0 & \begin{tabular}{|l|}
0.64592 \\
\end{tabular} & 0.0 & 0.0 & 7276 & 0.8 & 0.647369 & 28 & 5.1 \\
\hline $5: 3:$ & 23 & 21 & 17 & 2.13 & 0.49 & 0,0 & $0.6417 \pi / 2$ & 0,3 & fin & $80 \%$ & 140 & 0.638675 & 89 & 56 \\
\hline $6(a)$ & 30 & 24 & 19 & 105 & 2.53 & 0,3 & 2.362663 & 0.5 & 36.3 & $89 \%$ & -6.2 & 238495 & 09 & 72 \\
\hline $7(B)$ & 30 & 24 & 19 & 1.04 & 1.53 & 88 & 2,367156 & net & 38 & $72 \%$ & 2.3 & 2.372014 & f. & 39 \\
\hline $8: A$ & 36 & 27 & 22 & 0.29 & 2.31 & 0.1 & 4,061426 & 100 & 3.9 & $88 \%$ & 33 & 4.060335 & 13 & 7.1 \\
\hline $9\{5)$ & $3 E$ & 27 & 21 & 0.18 & 2.31 & 82 & $4.068: 7$ & D. & 2,0 & $90 \%$ & 2.3 & 4.656697 & 13 & 37 \\
\hline $20: A$ & 42 & 37 & 32 & 3.75 & 7.02 & 3,3 & 2.300612 & 27 & 72 & $96 \%$ & -3.5 & 2.742493 & $\sqrt{3} 3$ & 72 \\
\hline $213 j$ & 42 & 57 & 32 & 3.72 & 6.99 & $-0,1$ & \begin{tabular}{|l|}
2.0559 \\
\end{tabular} & 2.4 & 96 & $78 \%$ & $=8$ & 2.707589 & 17 & 81 \\
\hline $12: 4$ & 50 & 46 & 43 & 2.58 & 11.63 & 0.5 & 2.245024 & 2.0 & 3 & $48 \%$ & 3.7 & 2.414439 & 2.2 & 130 \\
\hline $23 / 3 ;$ & 50 & 46 & 43 & 2.65 & 11.58 & 1.8 & \begin{tabular}{|l|}
2.42832 \\
\end{tabular} & 13. & 49 & 9776 & -4.9 & 2.505366 & 2.1 & 205 \\
\hline
\end{tabular}

Figure 2-12 Q-Meter Calibration Initial Results

Figure 2-13 shows initial results of the repeatability of the Q-meter. The first set of data from Dec. 19 actually has a $150 \mathrm{~K}$ shroud setpoint. These results show relatively good repeatability from one cycle to the next, even when the external environment is different by $50 \mathrm{~K}$. There was some trouble with PI parameters for these runs, and the final repeatability measurements are expected to be even better.

\begin{tabular}{|c|c|c|c|c|c|c|c|c|c|}
\hline & & & & \multicolumn{3}{|c|}{ Results (w) } & \multicolumn{3}{|c|}{ Repeetablity ( $\mathrm{mw}$ ) } \\
\hline Step & TopT & Mlid T & Bet T & $19-\mathrm{Dec}$ & $21-D=c$ & 22-Dec & $\operatorname{ebs}(2-2)$ & $a b s(1-3)$ & ebs $(2-3)$ \\
\hline $1(2)$ & 18 & 15.5 & 24.5 & 0.382 & 0,380 & 0.332 & 12 & 2.1 & 2.3 \\
\hline $2\{a\}$ & 18 & 265 & 24.5 & 0,382 & 0.383 & 0.382 & 2.4 & 3.7 & 0.7 \\
\hline 32 & 20 & 18 & $1 E$ & 0.551 & 0.551 & 0.562 & 3.5 & 2.4 & 3.5 \\
\hline$A(3)$ & 20 & 18 & 15 & 0.562 & 0.554 & 0.563 & 2.3 & Q.4 & 1.4 \\
\hline $5(4)$ & 23 & 21 & 17 & 2,545 & 0.545 & $0.64 \overline{5}$ & 0.5 & 0.4 & 0,2 \\
\hline$E(B)$ & 23 & 21 & 17 & 0.642 & 0.644 & 0.642 & 2.2 & 1,0 & 2.2 \\
\hline
\end{tabular}

Figure 2-13 Q-Meter Repeatability Initial Results

Finally, the ability of the Q-meter to independently measure a heater power was also tested for a couple points during the initial calibration test. At a $36 \mathrm{~K}$ top temperature, $250 \mathrm{~mW}$ was applied, and it was measured to within 3 $\mathrm{mW}(1.2 \%)$. Additionally, at a $30 \mathrm{~K}$ top temperature, $50 \mathrm{~mW}$ was applied, and it was measured to within $1 \mathrm{~mW}$ $(2 \%)$.

The final and largest challenge uncovered in the initial Q-meter calibration test is that the Q-meter required much more heater power than initially expected. The next section details the issues that the thermal team had with the discrepancy between the available conductivity data, and the actual performance of the Aluminum 6061-T6 Q-meter at cryogenic temperature. 


\section{Q-Meter Conductivity Issue and Modification}

\section{A. Choice of 6061-T6 for central "spool"}

Aluminum 6061-T6 was chosen for two reasons:

1. 6061-T6 has a high conductivity at ambient temperature relative to its conductivity at cryogenic temperature. In the large ISIM Cryovac test, the Q-meters are in thermal series with the instruments. The Q-meter thus minimizes slowing the cool-down, while its reduced conductance at cryogenic temperature results in improved accuracy of heat load measurement.

2. Aluminum 6061-T6 is very commonly used in the aerospace industry, easy to machine, and its conductivity is well understood and documented. The team learned, however, that the conductivity of the material was not as well-understood as previously believed.

\section{B. Q-Meter Actual Conductivity}

During the first balance point, the Q-meter required far higher heater power on stages A and B than predicted. The first setpoint was predicted to require $357 \mathrm{~mW}$ on the top heater, and it balanced at $620 \mathrm{~mW}$. The following explanations were postulated:

1. The Q-meter was not completely pumped out, resulting in Free Molecular Heat Transfer between stages.

a. However, the Q-meter was not air-tight by any means, and temperature setpoints below approximately $27 \mathrm{~K}$ would have frozen nitrogen onto the Q-meter and reduced the pressure.

2. The nitrogen condensed on the Q-meter increased its emissivity from 0.1 to 1.0 .

a. Calculations showed the effect of an emissivity of 1 is a fraction of a mW. Additionally, the same factor was seen at temperatures above the sublimation point of Nitrogen at hard vacuum.

3. The Keithley heater supplies were reading incorrectly

a. The Q-meter was balanced against the cryocooler using different sets of heaters, and all resulted in cryocooler temperatures that matched the known capacity of the cryocooler.

4. The wires and Teflon insulation were contributing to the conductance

a. Hand calculations showed this contribution to be on the order of $\mathrm{mW}$.

5. The Q-meter was not actually made of 6061-T6 Aluminum.

a. Material certifications were consulted.

6. The Q-meter was not machined to the correct dimensions

a. Drawings were consulted and the other $4 \mathrm{Q}$-meter spools were measured.

After the above causes were investigated and subsequently rejected, the possibility that the 6061-T6 Aluminum in the Q-meter might not have the same conductivity as the trusted NIST data was considered. Two resources that more closely matched the results of the Q-meter were found in literature. The thermal conductance of the as-built Q-meter was measured by stepping through the temperature range with a difference in temperature of $1 \mathrm{~K}$ or $2 \mathrm{~K}$ between the Top (A) and Middle (B) levels of the Q-meter. Combined with precise knowledge of the area to length ratio from the very detailed $\sim 11,000$ node thermal model of the Q-meter, material conductivity was calculated from the measurements. Figure 3-1 shows test data, plotted with three conductivity resources for 6061-T6. Ball Aerospace (BASD) data, and data from a paper by Adam Woodcraft of the Cardiff School of Physics and Astronomy in the UK (Resource 5) much more closely match the conductivity of the Q-meter. The Q-meter data results from two separate runs with different PID heater parameters. Additionally, 2K DT datapoints are plotted at the extremes of the range and in the middle, which show good agreement with the $1 \mathrm{~K}$ DT datapoints. This is done to reveal any systematic errors in the temperature sensors. The datapoints at the bottom of the plot, corresponding to the secondary y axis indicate how well balanced each datapoint is. Most of these balances used sets of data whose best fit line's slope is lower than $1 \mathrm{~mW} / \mathrm{hr}$. 


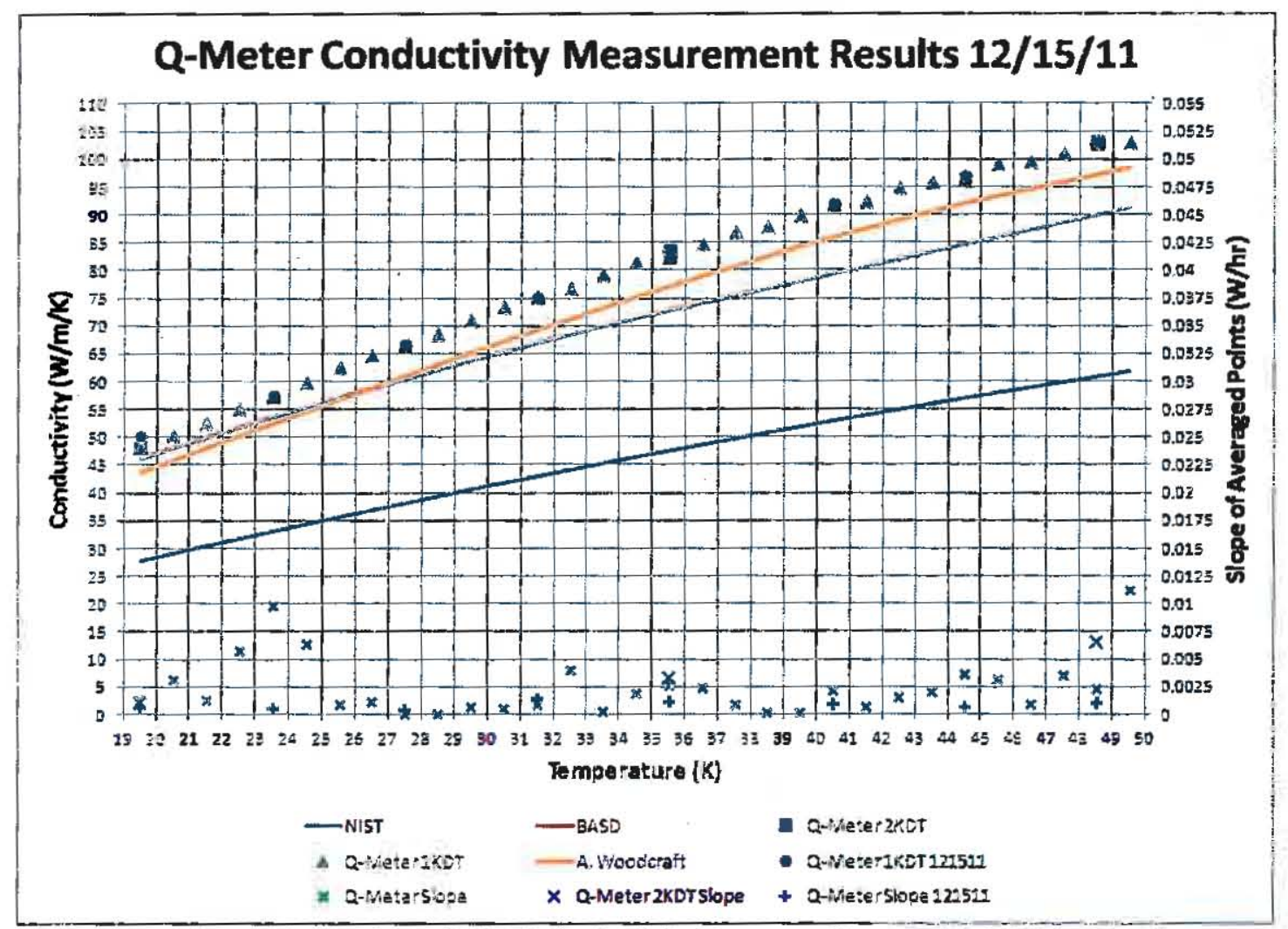

Figure 3-1 Aluminum 6061-T6 Conductivity Resources Compared to Q-Meter Results

The ISIM Thermal team's conclusion on this matter is not necessarily that the NIST data is incorrect. The paper by Adam Woodcraft (Reference 5) actually deals with this exact question. In his paper, Woodcraft states that significant variation in thermal conductivity may be observed in materials with even the same alloy and temper due to variations in the concentrations of impurities and tempering process that still fall inside the range allowed for the alloy. He goes on to say, "[M] ost thermal conductivity measurements were made several decades ago; changes in production methods may cause modern alloys to have different properties." Indeed, a paper (Reference 6) by scientists from NIST states that many of their cryogenic conductivity references have not been updated since 1977.

\section{Q-Meter Modification}

The Q-meter could have been left as-is. However, two consequences of failure to modify the Q-meters are:

1. The Q-meter inaccuracy due to long term drift of the accuracy of the Delta $T$ between the top (A) and middle (B) is directly proportional to the factor between the actual and expected conductance of the neck (see Reference 4 for more details).

2. The Q-meter would not have been able to reach some of the upper temperature levels that may have been required in the ISIM Cryo-vac test. While the temperature of the cold-plate interface on the Q-meter could be raised using the Q-meter warm-up heaters in the baseplate of the Q-meter during the calibration test, the refrigerator in the large Space Environment Simulator during the ISIM Cryo-vac test is much more powerful. Getting the top (A) and middle (B) temperatures to the upper portion of the initially planned range would have been impossible with the heater power designed into the Q-meters.

Besides the possibility of damaging the Q-meter, a concern with the modification of the Q-meter was that the modified Q-meter would slow down the test. The modified Q-meter would have a much lower cross sectional area in order to make up for the large difference in conductivity at cryogenic temperatures. However, although the Qmeter was not tested as such, conductivity values in the literature that differ by almost $80 \%$ at cryogenic temperatures differ only by $10-20 \%$ at room temperature. Thus, the modified Q-meter could slow the test in the 
initial stages of the cool-down. Fortunately, as Figure 3-2 shows, this is not the case. Figure 3-2 shows the predicted time in days for each of the JWST Scientific Instruments to reach a balance criterion during the Cryovac test. The simulation of the "As Built" Q-meters does decrease the time to balance by about 17 hours. However, modifying the Q-meter to match at cryogenic temperature had practically no effect on the slowest instrument when compared to the initially planned numbers. The reason for that probably has to do with the dominance of radiation at the beginning of the cool-down. Thus, when the difference in conductance between the planned Q-meter and the "Drilled Out" Q-meter is largest, the contribution of the straps (and Q-meters in series) is smallest.

\begin{tabular}{|c|c|c|c|c|c|c|c|c|}
\hline Name & $\begin{array}{c}\text { Criterion } \\
\text { (2)inr) }\end{array}$ & $\begin{array}{c}\text { Time isaches } \\
\text { ideris. }\end{array}$ & $\begin{array}{l}\text { Temperazure a: } \\
\text { Time Reachec is }\end{array}$ & $\begin{array}{c}\text { sa:e at Criterion } \\
\text { [K/nr) }\end{array}$ & \begin{tabular}{|c|} 
Temperature \\
Balarce $(K)$
\end{tabular} & $\begin{array}{c}\text { Ttoss, } \\
\text { Batanceiki }\end{array}$ & \begin{tabular}{|c|} 
Rate at \\
Ealarce KKinhi
\end{tabular} & $\begin{array}{c}\text { Steacy State } \\
\text { Temperat re is: }\end{array}$ \\
\hline NHSDEC Bench Qmeter As S annes & 0.0244 & 24.22 & 38.86 & -0.0112 & 38.24 & 0.24 & -0.0020 & 3329 \\
\hline Nis5गEC FDA Qmeter is Planned & 5.3979 & 2.50 & 36.49 & -6.2510 & 35.45 & -0.05 & 0,0000 & 3543 \\
\hline NikCam bench Qmater A3 Plannoc & 0.0855 & I.08 & 39.52 & -0.0650 & 38.51 & 0.02 & $\operatorname{coccio}$ & 32.52 \\
\hline FG5 Qmetar As Planped & 0,0056 & $18, \pi 3$ & 38.75 & -0.0030 & 38,73 & -2.05 & -0.0030 & 33.79 \\
\hline 5x. Avg Qmeter As Dlahnes & 2.3 & & & & 42.24 & 0.80 & -0.0230 & 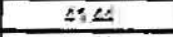 \\
\hline NifsoeC Eerch Qneter A5 bult: & 0.0144 & 23.67 & 38.87 & 20.40 & 33.25 & 0.05 & -0.0020 & 33.22 \\
\hline N/RSPEC FPAO Oneter AS SWil: & 0.3979 & 11.88 & 36.49 & -0.2300 & 36.43 & -0.25 & 0.2000 & 32.26 \\
\hline Nifiam berch Qmater is Bufl: & 0.0883 & 22.17 & 39.70 & $-6.0 \overline{0}$ & 38.52 & 0.22 & $0, \cos x$ & 3350 \\
\hline FoS Qme:Er A3 bult! & 0.0036 & 15,94 & 38.73 & -0.2050 & 33.73 & -0.55 & -0.0030 & 35.79 \\
\hline Sir. Ave Qmeter as Sult & 0.5 & & & & 42.30 & 0.86 & 0,0240 & 49.2 \\
\hline WhS PEC Sench Q-meter Drilled O O. & 0.0524 & 24.42 & 39.87 & -0.0140 & 3825 & 2.05 & -0.0020 & 3320 \\
\hline NASPEC =DA Qmezer arilled Ou: & 0.3979 & 12.30 & 36.49 & -0.2370 & 36.2 .5 & -0.05 & 0.0000 & 3545 \\
\hline NiFCam Eancin Qmeter Drillac OL? & 0.0825 & 23.08 & 39.65 & -60800 & 38.51 & 2.02 & 0.0000 & 3552 \\
\hline FGs ameter Drilled OUt: & 0.0038 & 18.72 & 38.73 & -0.6030 & 38.73 & -2.5 & $-0 . \cos \theta$ & 3579 \\
\hline Str. Ave Qneter Drifined OW: & 0.5 & & & & $\therefore 2.27$ & 0.83 & -0.0230 & sill \\
\hline
\end{tabular}

Figure 3-2 Effect of Q-meter Modification on Cryovac Test Cool-Down

Therefore, it was decided to drill out the center of the Q-meters in order to match the initially planned conductance. Figure 3-3 shows the conductance factor as a function of the average temperature of the neck between the top (A) and middle (B) stages of the Q-meter. Since the Q-meter is primarily planned to operate in the range from $30-42 \mathrm{~K}$, the datapoints in that range were averaged, and a final result of 1.74 was obtained. This conductance factor became the area reduction factor.

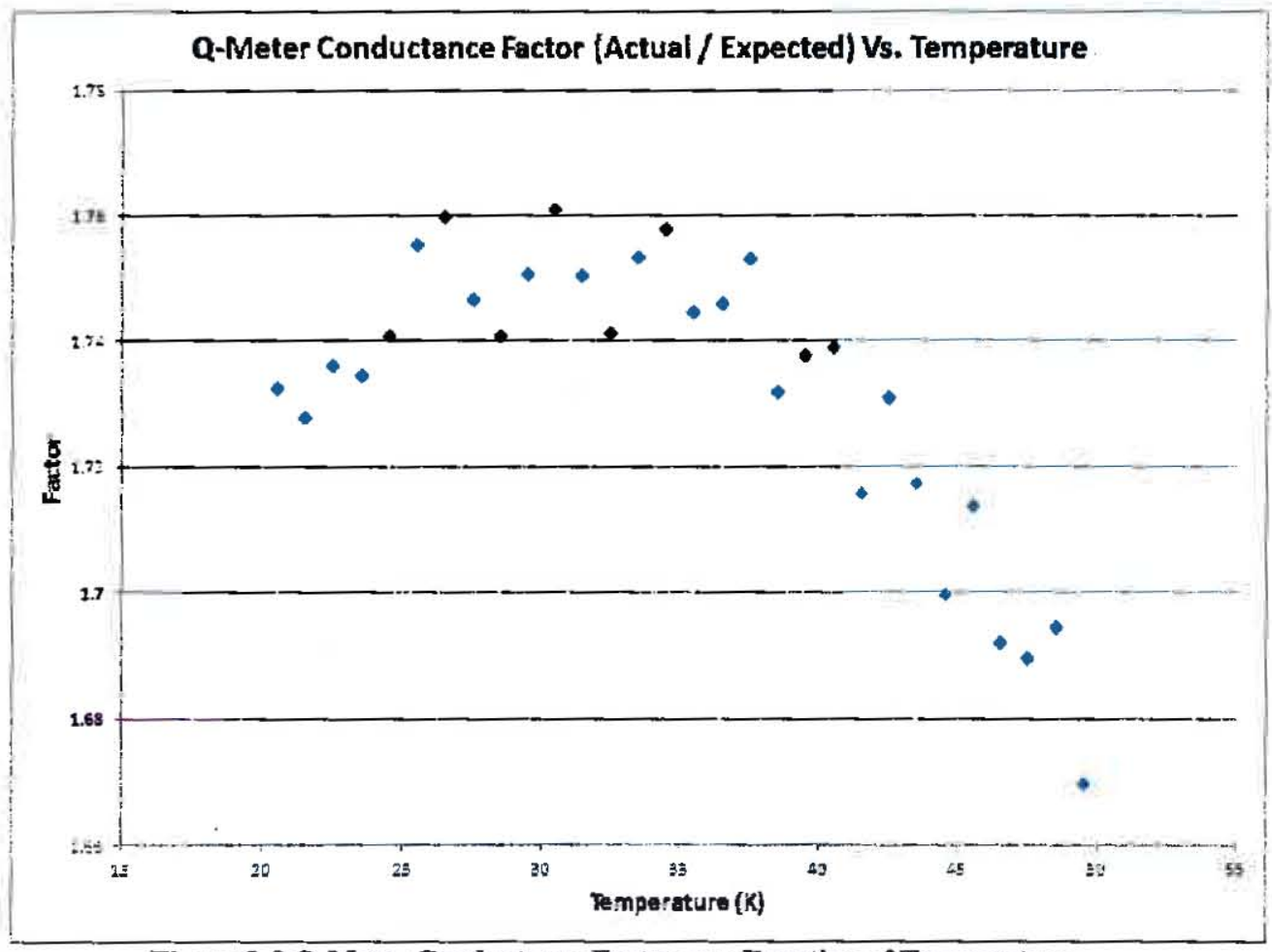

Figure 3-3 Q-Meter Conductance Factor as a Function of Temperature

17

American Institute of Aeronautics and Astronautics 
Figure 3-4 shows the plan for drilling out the center of the Q-meter. Standard hole sizes were chosen that most closely matched the area reduction goal. The holes in red may look very large, but they are actually only reducing the area by $43 \%$. Approximately $57 \%$ of the cross sectional area remains. Figure $3-5$ shows the $\sim 11,000$ node Thermal Desktop model results after the modification. Especially with respect to the top (A) heater power, the expected power (from original NIST data) matches the new power (with actual conductivity, drilled out) very closely. One side effect of the drilled hole is that the top of the Q-meter gains a radiative view of the bottom. Close inspection of the data in Figure 3-5 reveals this effect. When the top heater powers are compared between calibration and the Cryovac Chamber (SES), the only temperature difference is the bottom level. It is colder in the SES chamber. This seems to result in a fraction of a $\mathrm{mW}$ difference in top heater power, because of the view to the bottom. Therefore, a small disc of aluminum tape was taped on both sides of the central hole, and staked with stycast. Figure 3-6 shows this modification.

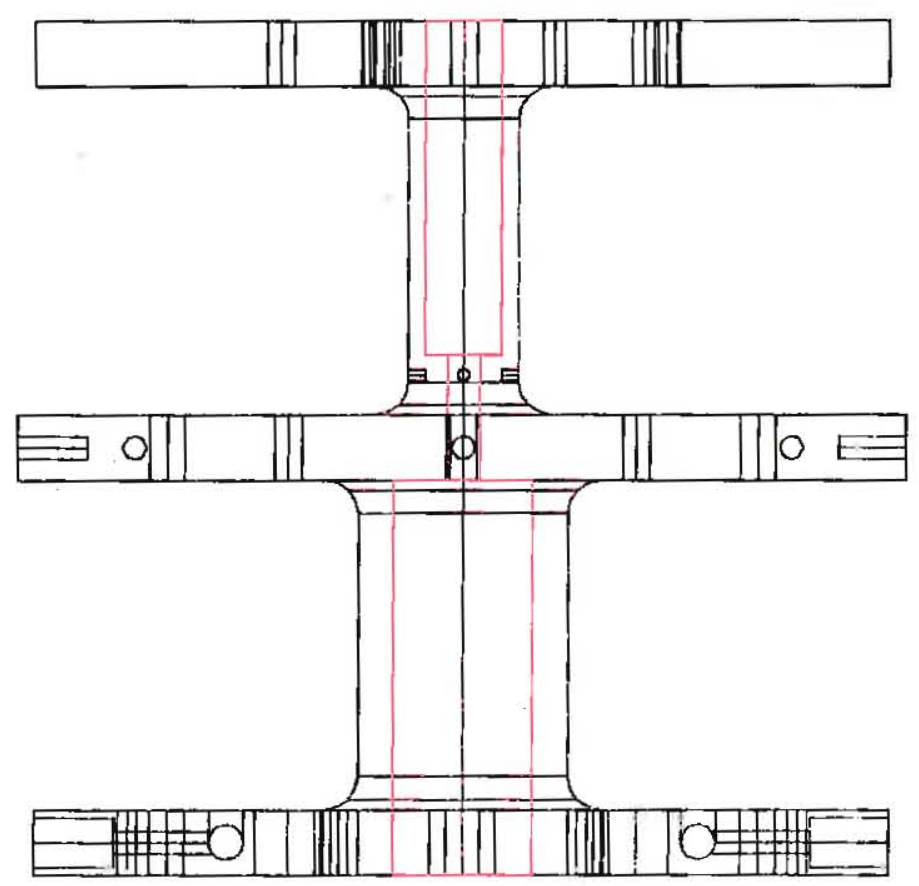

Figure 3-4 Q-Meter Area Reduction Holes

\begin{tabular}{|c|c|c|c|c|c|c|c|}
\hline & Tos Temperwiure ik & |Fidcle Tenperature (K) & Bonom Tis; & Expocted Top aiste & 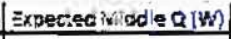 & Naw Top Q Model Predict: & New Middle Q Model Predict \\
\hline \multirow{8}{*}{ 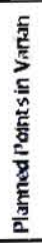 } & 13.00 & 27.4 & 15.49 & 2090 & 0.055 & 0.8894 & 0.2762 \\
\hline & 19.00 & 18.0 & $2 E .63$ & 0.955 & 0.152 & 0.1564 & 0.2783 \\
\hline & $2 x \cdot x$ & 28.5 & 16.70 & 2245 & 0.293 & 0.2450 & 0.3421 \\
\hline & 30.02 & 25.6 & 19.12 & 8002 & 1.981 & 1.0228 & 2.8937 \\
\hline & 36.00 & 30.0 & 21.17 & $458 ?$ & 3.214 & 2.6157 & 2.9006 \\
\hline & 46.20 & 32.4 & 21.69 & $242 ?$ & $2.69 \AA$ & 2.4645 & 2.5085 \\
\hline & 45.00 & 33.2 & 22.50 & 3530 & 2.514 & 3.6096 & 1.9024 \\
\hline & 56.00 & 36.5 & 24.52 & 4433 & 2.357 & 4.3689 & 2.3150 \\
\hline \multirow{9}{*}{ 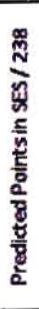 } & 3300 & 28.0 & 4.8 & $=$ & - & 0.1566 & 3.0948 \\
\hline & 30.00 & 25.6 & 18.0 & - & -- & 1.0228 & 2.3600 \\
\hline & 36.50 & 30.0 & 28.0 & - & $\cdots$ & 1.6158 & 4.3124 \\
\hline & 40.60 & 31.2 & 280 & - & $\cdots$ & 2.4647 & 4.1748 \\
\hline & 45.00 & 35.2 & 13.0 & - & $\approx$ & 3.6098 & 3.9212 \\
\hline & 47.50 & 32.5 & 18.0 & - & $=$ & 4.7300 & 2.3674 \\
\hline & 48.00 & 33.0 & 18.6 & $=$ & $=$ & 4.6714 & 2.4904 \\
\hline & 49.00 & 34.5 & 18.2 & - & - & 4.5593 & $3.6 \pi 04$ \\
\hline & 50.20 & 35.5 & 18.0 & - & - & 4.3692 & 5.4747 \\
\hline
\end{tabular}

Figure 3-5 Post-Modification Predictions 


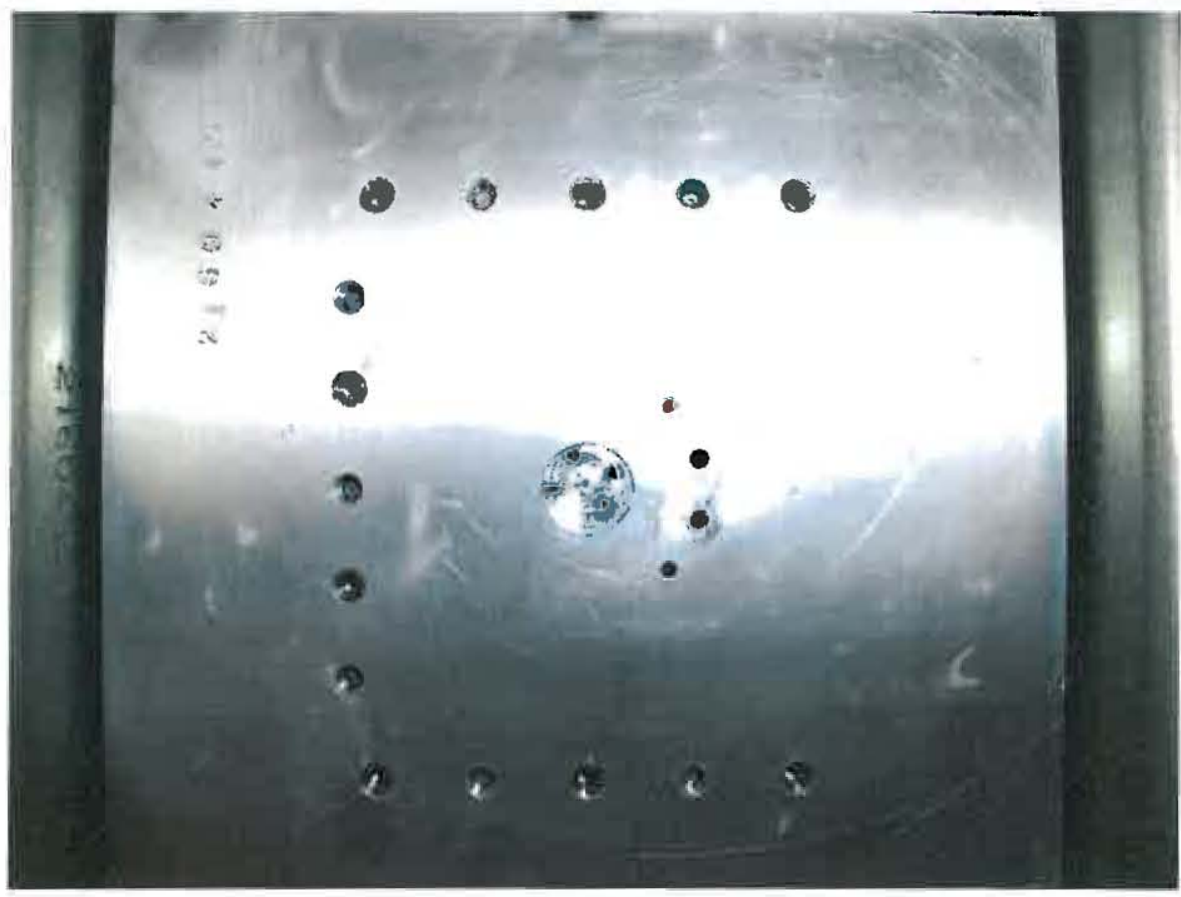

Figure 3-6 Q-Meter Area Reduction Holes

\section{Calibration Re-Test Results}

After the modification of the Q-meter, heater power required at each stage matched matched the initial predictions to within $3 \%$. The calibration test was able to reach all setpoints and balance to better than $1 \mathrm{~mW} / \mathrm{hr}$ at each, with similarly small standard deviations and ranges. Each of these datapoints is the result of two "steps" in the Labview program. The first step utilizes high gains in the PI controller to get very close to balanced with the setpoints required. At that point, the gain is dropped and the integral is kept the same. This forces the program to decrease the magnitude of its adjustments to the 1 or $2 \mathrm{~mW}$ range. The program waits until its stability criteria are met, and then dwells for 30 minutes. As was done for the initial calibration testing, a Visual Basic program was used to march through the data and perform statistics on 300 datapoints (10 minutes) at a time throughout the range, choosing the best point in the dwell to report. Figure 4-1 shows a small subset of the data collected during the recalibration testing of the Q-meter after the area modifications.

\begin{tabular}{|c|c|c|c|c|c|c|c|c|c|c|c|c|}
\hline & \multicolumn{5}{|c|}{ Nonitor } & \multicolumn{2}{|c|}{ Prediction } & \multicolumn{5}{|c|}{ 3es: 300} \\
\hline Step & TosT & Mid T & $30: T$ & Mid Q & $\operatorname{sot} \alpha$ & $\operatorname{Tog} Q$ & $\%$ Off & slope & Average & stder & Eange & $520:$ \\
\hline $2(A)$ & 18 & 16.8 & $24.4 E$ & 0.09 & 0.97 & 0.1720 & 267 & $a, a$ & 0.2695 & 04 & 23 & $100 \%$ \\
\hline $4[B]$ & 18 & 16.8 & 24.48 & 0.09 & 0.96 & 0.1740 & 1.96 & -0.3 & $0,2 \pi 7$ & 2.7 & 27 & $72 x_{2}$ \\
\hline $14\{A\}$ & 30 & 25.5 & 27.8 & 2.72 & 1,78 & 1.0370 & 3.13 & 0.0 & 10053 & 9.3 & 21 & EA\% \\
\hline $15\{B\}$ & 30 & 23.5 & 17.82 & 1.72 & 1.72 & 1.0370 & 3.15 & 0.5 & 1.0053 & 0.4 & 27 & $38 \%$ \\
\hline $52(4)$ & 36 & 30.4 & 20.47 & 294 & 2.32 & 1.3050 & 2.91 & 0.0 & $2462 j$ & 0.3 & 2.1 & $E 7 \%$ \\
\hline $64(3)$ & 36 & 30.4 & 20.47 & 295 & 2.31 & 1.5050 & 3.02 & -0.2 & 1.4621 & 0.4 & 2.5 & $95 \%$ \\
\hline $225(4)$ & 40 & 31.8 & 20.58 & 2.87 & 1.26 & $2.3<70$ & 2.87 & 013 & 2.2814 & 98 & 3.6 & 9836 \\
\hline $128(5)$ & 40 & 31.2 & 20.57 & 2.88 & 1,27 & 2.3470 & 2.84 & 82 & 2.2823 & 9.3 & 4.0 & $38 \%$ \\
\hline $274 \Delta$ & 50 & 35.6 & 22.5 & 1.76 & 1.52 & 4.5620 & 2.59 & -0.8 & 4.5404 & 1.2 & 5.7 & $83 \%$ \\
\hline $276(5)$ & 50 & 35.6 & 22.51 & 1.78 & 1.47 & $26 E 20$ & 2.52 & a.2. & 4.5473 & 2.3 & 8.8 & $77 \%$ \\
\hline
\end{tabular}

Figure 4-1 Post Modification Calibration Testing Results

At this point in the testing, completion of the calibration through all 4 cycles was interrupted by an overtemperature event on the hardware. During unattended operation over a weekend, the chamber experienced a pressure spike, resulting from nitrogen ice sublimation as the chamber warmed through the $25-30 \mathrm{~K}$ temperature range. The pressure spike triggered the Labview software's manual mode, which shut down cooling but failed to 
turn off internal Q-meter heaters. The Q-meter reached a temperature of approximately $500 \mathrm{~K}$, which required replacement of all heaters, sensors, and wires and the chamber's cryocooler.

\section{Conclusions and Lessons Learned}

\section{A. Conclusions}

The calibration testing of the Q-mieters designed for use in the Cryo-vacuum testing of the Integrated Scientific Instrument Model of the James Webb Space Telescope at Goddard Space Flight Center has shown the Q-meters to be a quite capable and robust system. The development of these devices results in a system that is well characterized, and will perform as-designed. The ISIM team anticipates that these 5 Q-meters will be used repeatedly at Goddard for even more applications than are currently envisioned. In fact, the first use is planned for mid July 2012, when the first calibrated Q-meter will be used as a stand-in for a cryocooler stage in the MIRI shield balance test, at temperatures as low as $18 \mathrm{~K}$.

\section{B. Lessons Learned}

For the benefit of future attempts at building and calibrating accurate cryogenic Q-meters, the authors have compiled the following list of lessons learned:

- Almost any metal can exhibit conductivity characteristics that are difficult to predict, especially alloys, and especially at cryogenic temperatures. Always plan to measure conductivity of the material in question if the property is critical to the application. Additionally, as even batches of 6061-T6 that are $74 \%$ different in conductivity at cryogenic temperatures are only $10 \%$ different at ambient temperature, the testing must be done at the final desired temperature.

- For critical applications, never trust just one or two sets of property data, even if they are very highly trusted and accepted as the official properties by the project.

- For applications in which conductance is critical, either design hardware that works for all published conductivities, or retain the ability to remove material if needed.

- Any highly-accurate Q-meter must be pre-calibrated. Reliance on property data only would have produced a useless instrument.

- Always incorporate both hardware and software safety limits for the test article, even if it is non-flight, especially during unattended operation. The exact details of the event are beyond the scope of this paper, but the test experienced an over-temperature condition during unattended operation that resulted in damage to the Q-meter and test facility, requiring extensive refurbishment. The computer responded to a pressure spike by turning off cooling, but not heating, and also disabled the over-temperature protection in the software. A secondary system using the Lakeshore 218 units may have prevented the need for refurbishment.

- Dale-Ohm heaters, CERNOX sensors, and Diodes have been very robust during the calibration test, and have proven their worthiness for this application.

- Be sure to thermally sink all harnessing that crosses thermal boundaries.

- Aluminum tape or thermally sunk foil is a much better boundary to thermal radiation (see application on nitrogen shroud) than even thick blankets. 


\section{Appendix - Acronyms}

$\begin{array}{llll}\text { FGS } & \text { Fine Guidance Sensor } & \text { MIRI } & \text { Mid Infrared Instrument } \\ \text { GHe } & \text { Gaseous Helium } & \text { NIRCam } & \text { Near Infrared Camera } \\ \text { GN }_{2} & \text { Gaseous Nitrogen } & \text { NIRSpec } & \text { Near Infrared Spectrograph } \\ \text { GSE } & \text { Ground Support Equipment } & \text { OSIM } & \text { OTE Simulator } \\ \text { GSFC } & \text { Goddard Space Flight Center } & \text { OTE } & \text { Optical Telescope Element } \\ \text { ISIM } & \text { Integrated Science Instrument Module } & \text { PI } & \text { Proportional Integral Controller } \\ \text { JWST } & \text { James Webb Space Telescope } & \text { SES } & \text { Space Environment Simulator } \\ \text { LN }_{2} & \text { Liquid Nitrogen } & \text { SI } & \text { Science Instrument }\end{array}$

\section{Acknowledgments}

The authors would like to thank Dr. J. Tuttle, Mr. M. Kobel, Mr. D. McGuffey, Mr. M. Martins, and Mr. P. Cleveland for valuable inputs into the design and calibration test configuration, as well as Mr. J. Roddin for detailed design models and drawings and Dr. S. Courts for his collaboration on choosing the correct temperature sensors.

\section{References}

${ }^{1}$ Parrish, K., Glazer, S., Thomson, S., "The Cryogenic Thermal System Design of NASA's James Webb Space Telescope (JWST) Integrated Science Instrument Module (ISIM)", ICES Conference, Paper 05ICES-206, 2005

${ }^{2}$ Glazer, S., Cleveland, P., "James Webb Space Telescope Integrated Science Instrument Module Thermal Balance/Thermal Vacuum Test Configuration and Test Planning at NASA's Goddard Space Flight Center", ICES Conference 2010

${ }^{3}$ Parrish, K. and Cleveland, P., "Thermal System Verification and Model Validation for NASA's Cryogenic Passively Cooled James Webb Space Telescope (JWST)", ICES Conference, Paper 05ICES-236, 2005

${ }^{4}$ Comber, B., Glazer, S., and Cleveland, P., "James Webb Space Telescope Integrated Science Instrument Module Design, Optimization, and Calibration of High-Accuracy Instrumentation to Measure Heat Flow in Cryogenic Testing" , $41^{\text {st }}$ ICES Conference, Paper AIAA-2011-5009, 2011

${ }^{5}$ Woodcraft, A., "Predicting the Thermal Conductivity of Aluminum Alloys in the Cryogenic to Room Temperature Range" , Cryogenics 45(6): 421-432, 2005.

${ }^{6}$ Marquardt, J.P. Le, and Radebaugh, Ray, "Cryogenic Material Properties Database", $11^{\text {th }}$ International Cryocooler Conference, 2000. 